\title{
Correlations between IL6 and the main clinical and biological parameters in rheumatoid arthritis
}

\author{
Mihaela Chicu ${ }^{1}$ Eugen Bitere ${ }^{2}$, Gabriela Dogaru ${ }^{3}$ \\ 1. Pharmacy "Gr Popa "Iasi, Department of Pathophysiology; „St. Spiridon” County Hospital Iasi \\ 2. Institute of Cardiovascular Diseases "Prof. Dr.G. IM. Georgescu "Iasi, Department of Cardiovascular Surgery \\ 3. Clinical Rehabilitation Hospital Cluj-Napoca, University of Medicine and Pharmacy Cluj-Napoca
}

Mihaela Chicu, e-mail: mihaielabitere@yahoo.com

\begin{abstract}
Introduction: Cytokines are a family of complex peptide with hormone-like activity. They are soluble proteins without enzymatic activity and serves as the main intracellular mediators. Many cytokines achieves its effects by binding to special receptors membrane, and their adjustment is via soluble receptors. Cytokines are characterized by pleiotropism, overlapping and mutual adjustment. Proinflammatory cytokine involved in major rheumatoid arthritis are TNF, IL1 $\alpha$, IL1 $\beta$, IL8. The biological effects of IL6 overlap in large part over those of TNF. If TNF is involved in induction of apoptosis or programmed cell death, IL6 is specifically associated with angiogenic factors activation and the occurrence of neovascularity to the synovium; favors articular cartilage degradation by increasing the release of MMP, decreasing PG, recruit osteoclasts, apoptosis of osteoblasts, release of degradative enzymes and the inflammatory mediators - iNOS, COX2 - TNF, IL6, IL8.Material and methods: Based on these data we proposed and realized - for the first time in Romania - the measurement of IL6 levels and the correlation with values of DAS28 score, HAQ, ESR, CRP, Hb and the immunological parameters too. The study was conducted on a group of 80 sick diagnosed with RA in various stages of evolution, under treatment with disease-modifying medication , type Methotrexate, Arava.Conclusions: Levels of IL-6 correlate a direct manner with those of acute phase reactants ,ESR, CRP and indirect values of $\mathrm{Hb}$, IgG; the clinical parameters (number of tender and swollen joints, DAS28, HAQ) are not influenced by values IL6.
\end{abstract}

Keywords: IL6, DAS28, HAQ.

\section{Introduction}

Rheumatoid arthritis, found in literature as the evolutionary chronic polyarthritis too, is a desease characterized by chronic infiltrative-proliferative inflammation of the synovial in diarthrodial joints. It is clinicaly expressed by the presence of arthritis in many joints.

The etiology of rheumatoid arthritis is unknown. As other chronic rheumatic or non-rheumatic diseases, the etiology it is probably multifactorial, in which environmental factors interact with a susceptible genotype.

Genetic susceptibility is argued by familiy studies and correlation with histocompatibility antigens. Family aggregation suffering with other related diseases or with immune abnormalities (with or without clinical expression) is 
significantly higher than that found in members of witness families. Reffering to HLA, DR4 and DR1 types were more prevalent in thick patients. Looking to environmental factors, the most importants are those infectious. Over time they were discussed multiple bacteria, microplasma, viruses.
In RA, macrophages have a high activity in secretion of various inflammatory cytokines, the most important being: the tumor necrosis factor (TNF), interleukin 1 (IL-1), interleukin 6 (IL-6), and increasing amounts of prostanoids too .

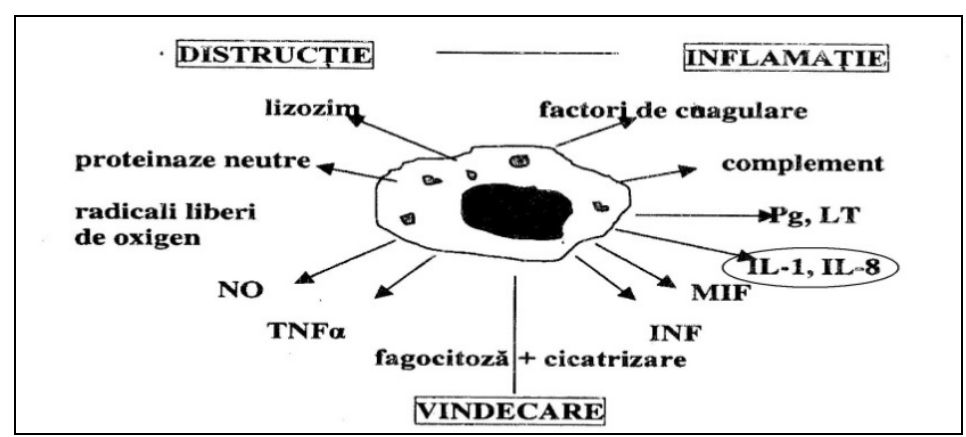

Fig. 1. Secretory function of macrophages in inflammation

The main proinflammatory cytokine with a major role in RA are represented by TNF, IL1 $\alpha$, IL-1 $\beta$, IL6, IL8.

IL6 is the prototype of the proinflammatory cytokine and has several biological properties which act in the sense of favoring inflammation. The most important property of this kind is its ability to initiate and sustain expression of cyclooxygenase-2 (COX-2) and the nitric oxide inducible synthesis (iNOS). This explains the large amount of PGE2 and NO produced by cells exposed to IL6.

Another important pro-inflammatory property of IL6 is to increase the expresion capacity of adhesion molecules, such as ICAM-1, on surface of endothelial cells and other cells. This promotes the infiltration of inflammatory and immunocompetent cells in the extravascular space. IL6 was particularly associated with activation of angiogenic factors that contribute to neovascularization. In addition IL6 stimulates and promotes cartilage destruction by rapid induction of proteoglycan loss and stimulating the production of proteinase in chondrocytes.

Material and methods: Based on these elements, the study has proposed and realized - for the first time in our country the measuring of the IL-6 levels in women patients with RA in various stages of evolution and different disease onset age, and their correlation with values of the main clinical parameters (NAD, NAT, DAS28, HAQ score) and laboratory (ESR, CRP, IgG).

Data from the study were processed using medical statistics programs SPSS (version 13.0), EPIINFO - version 6 and Excel XP. The study of the correlation between various events was carried out by using the correlation coefficient " $\mathrm{r}$ " (Pearson) that reveals the statistical links intensity and their meaning. The correlation coefficient values are in the range $(-1,+1)$ : 
- if the correlation coefficient tends to +1 , there is a very high linear dependence phenomena, direct correlation;

- if the correlation coefficient tends to -1 , there is also a very high dependence phenomena, inverse correlation;

- if the correlation coefficient is closer to 0 , the more less is theintensity of the relationship.

The kit used to determine IL6 was produced in the US ( $\mathrm{R} \& \mathrm{D})$ and was purchased from the company Cyber-med. Samples were worked in the Department of Immunology of UMF Iasi.

Results: The whole group of 80 patients with RA, a percentage of $82.5 \%$ was under the age of 60 years when the disease started and only $17.5 \%$ had more than 60 years. Between the two study groups, IL6 values presents no statistically significant differences.

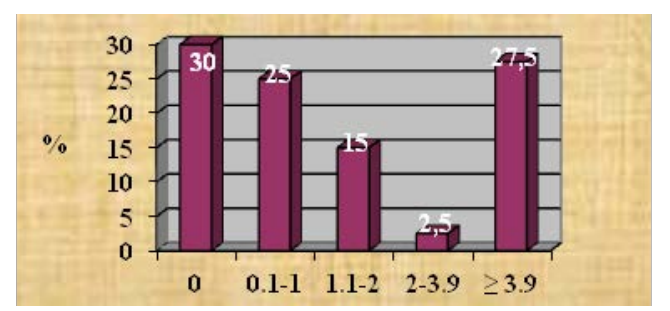

Fig. 2. IL6 distributions of the studied group

The two study groups presents no obviuous statistical differences of the IL6 levels ( $\chi 2$ $=0.86 ; \mathrm{GL}=2, \mathrm{p}=0.65$ ).

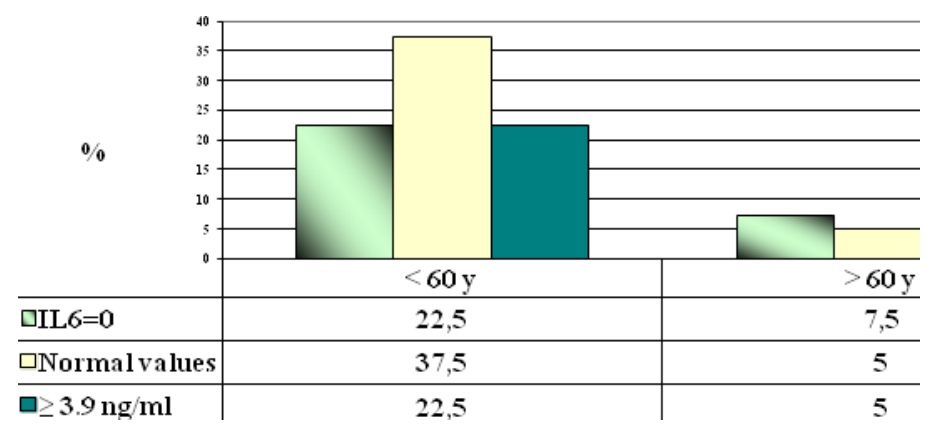

Fig. 3. The distribution of patients by age according with $I L-1 \beta$ levels

Trying to highlight the influence of this immunological parameter on the development of inflammatory process, we analyze the correlations between IL6 levels and clinical, functional, biological and radiological parameters.
Correlating the number of tender or swollen joints with IL6 levels is observed that these clinical parameters are independent of the IL6 values $(r=0.06$,

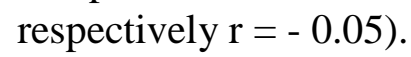



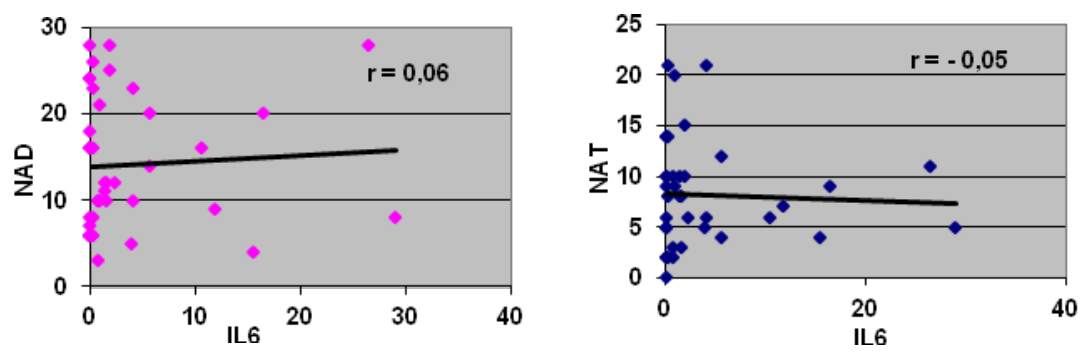

Fig. 4. Correlation between IL6 and the number of tender and swollen joints of patients in the study group

Most of the IL-6 values being close to 0 or normal $(<3.9 \mathrm{ng} / \mathrm{ml})$, it is noted that the number of tender joints increases with higher values of interleukin, while the number of swollen joints has a tendency to indirect correlation.

The correlation between IL6 levels and DAS28 score is direct and the dependence of IL6 on the score is in a 13\% ratio.

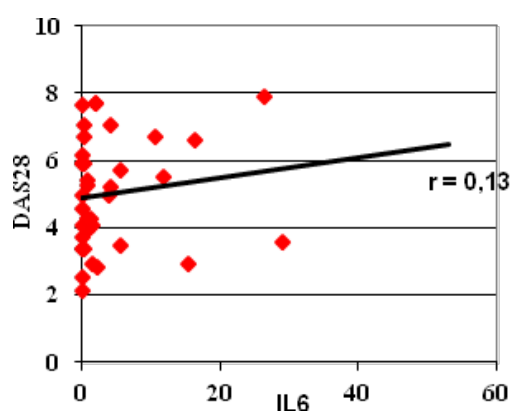

Fig. 5. Correlation between IL6 levels

and DAS28 score in patients

of the study group

Between IL6 levels and HAQ score exists a direct correlation, but IL6 influence on score value is not important $(r=0.08)$.
Fig. 6. Correlation between HAQ score and IL6 levels in patients

in the study group

An average direct correlation was obtained between the IL6 levels and ESR values ( $\mathrm{r}=$ $0.30)$.

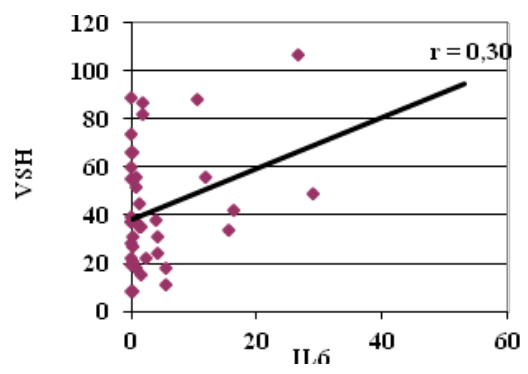

Fig. 7. Correlation between IL6 levels and ESR values in patients in the study group

If the levels of IL-6 increases, then CRP values increases too, that shows a direct correlation $(\mathrm{r}=0.41)$.

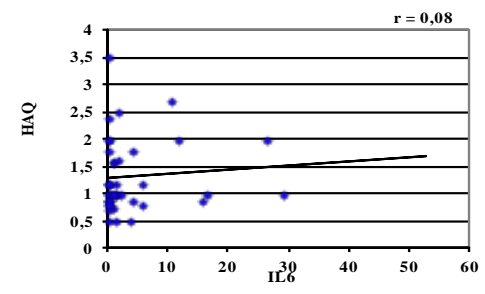




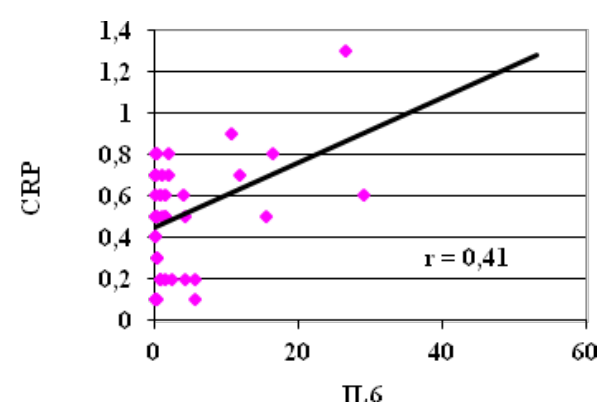

Fig. 8. Correlation between IL6 levels and CRP values in patients in the study group

Correlation between the values of IL6 with $\mathrm{Hb}$ levels is statistically indirect, pointing out that the high levels of interleukin $1 \beta$ correspond to low hemoglobin levels $(r=-0.50)$.

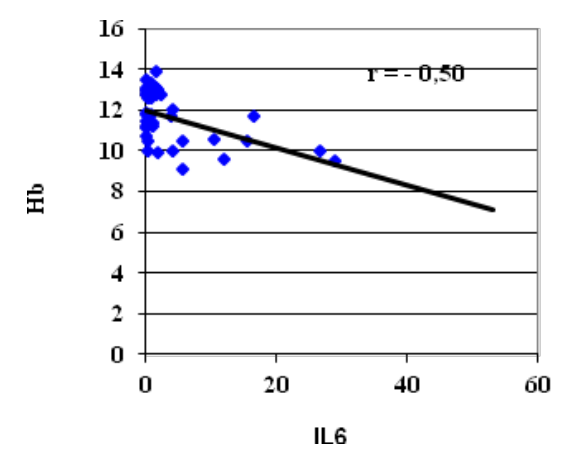

Fig. 9. Correlation between IL6 levels and $H b$ values in patients in the study group

Correlation between IL6 levels and IgG values is indirect and insignificant from statistic point of wiew $(r=-0.13)$.

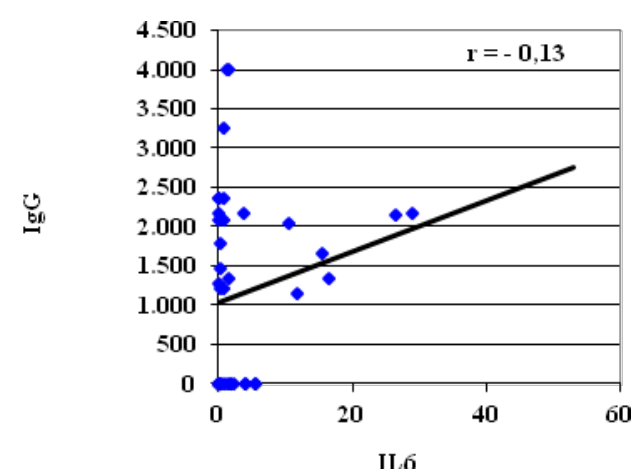

Fig. 10. Correlation between IL6 levels and IgG values in patients

in the study group

L6 levels presents an insignificant indirect correlation with titer values of Latex and Waaler-Rose reactions.
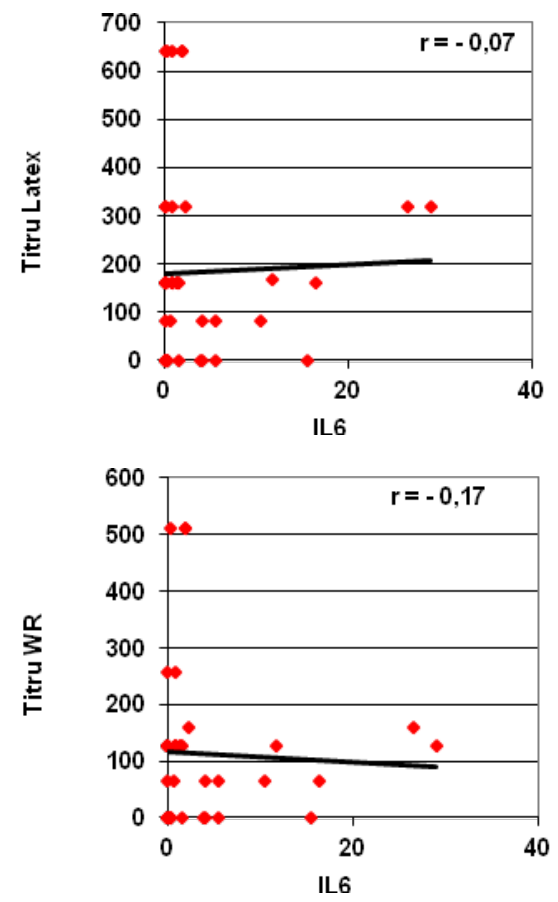

Fig. 11. Correlation between IL6 levels and Latex and Waaler-Rose reactions titers values in patients in the study group

\section{Conclusions:}


IL6 levels in RA correlate in a direct manner, statistically significant with NAD, DAS28 and HAQ scores, ESR and CRP, and in an indirect manner with $\mathrm{Hb}$ values, IgG, and Waalter-Rose Latex reactions.

\section{Bibliography:}

Dejica D: Rheumatoid Arthritis. Clinical Immunology Treaty, vol; Ed. Dacia, 1997; 278-309;
Deveraux QL; Reed JC: IAP family proteins - suppressors od apoptosis, Genes Dev., 1999.13: 491-96;

Dinarello CA. - The role of IL-1 receptor antagonist in blocking inflammation mediated by IL-1. N Engl J Med, 2000, 343-349;

Dinarelo C.A., L.L. Moidawer Proinflammatory and anti-inflammatory cytokines in rheumatoid arthritis - a primer for clinicians; 2nd ed. Thousand Oak, CA: Amgen Inc; 2000. 